\title{
Commentary \\ The simple and the simpler in pneumonia diagnosis
}

\section{Thiago Lisboa and Jordi Rello}

\begin{abstract}
Critical Care Department, Joan XXIII University Hospital. University Rovira \& Virgili, Institut Pere Virgili, CIBERes Enfermedades Respiratorias, Carrer Mallafre Guasch, 4 - 43007,Tarragona, Spain
\end{abstract}

Corresponding author: Jordi Rello, jrello.hj23.ics@gencat.net

Published: 15 June 2007

This article is online at http://ccforum.com/content/11/3/140

(c) 2007 BioMed Central Ltd

Critical Care 2007, 11:140 (doi:10.1186/cc5933)

See related research by El Solh et al., http://ccforum.com/content/11/3/R57

\begin{abstract}
In the absence of a perfect 'gold standard' for diagnosing pneumonia, comparing diagnostic performance between techniques remains controversial. El Solh and coworkers present a study evaluating use of quantitative endotracheal aspirate culture to enhance diagnostic accuracy in pneumonia patients admitted from nursing homes. We discuss the use of quantitative cultures and thresholds to differentiate between colonization and infection in pneumonia patients; we also consider the inaccuracy of diagnostic studies, which compromises the reproducibility of these data in clinical practice.
\end{abstract}

El Solh and coworkers [1] present an interesting study in which they evaluated use of quantitative endotracheal aspirate (QEA) cultures as a diagnostic tool for determining presence of pneumonia in ventilated patients admitted from nursing homes. The 2005 American Thoracic Society (ATS)/ Infectious Disease Society of America (IDSA) guidelines [2] recommend that the approach to such patients should be like that in patients with ventilator-associated pneumonia (VAP). One of the strengths of this study is that it evaluates this specific population. The study compared the diagnostic accuracy of QEA with that of protected specimen brush (PSB) and bronchoalveolar lavage (BAL). Unfortunately, the impact of the findings on antibiotic therapy and patient outcomes was not assessed. Other potential limitations not discussed in the report include the lack of evaluation of QEA sample quality, which is a major problem in interpretation of study findings [3], and failure to consider the influence of comorbidities such as chronic obstructive pulmonary disease (COPD).

The study shows that bacterial counts obtained through QEA correlate with those obtained by PSB $(r=0.71)$ and BAL $(r=0.77)$. However, Table 2 highlights an important weakness of aetiological diagnosis based on a threshold;
Staphylococcus aureus was isolated in 50\% more cases in BAL samples as compared with PSB samples, using the recommended threshold. Furthermore, the authors constructed receive operating characteristic (ROC) curves to evaluate the diagnostic accuracy of QEA, and found a sensitivity of $90 \%$ and a specificity of $77 \%$ when $10^{4}$ colony-forming units $/ \mathrm{ml}$ was used as the diagnostic threshold. The threshold of $10^{4}$ colony-forming units $/ \mathrm{ml}$ is lower than thresholds recommended by IDSA/ATS [2] to differentiate colonization from infection in QEA cultures. Therefore, what threshold should be used at the bedside?

Use of bacterial counts as a diagnostic threshold in pneumonia has been proposed to differentiate colonization from infection [4]. It aims to avoid over-use of antibiotics in patients with low bacterial counts on quantitative cultures, defining a cut-off for pneumonia diagnosis. The main concern about defining such a cut-off is methodological. The ROC curve is a useful tool for evaluating performance and accuracy of diagnostic tests, but construction of ROC curves assumes that there is a gold standard $[5,6]$. In the absence of a gold standard, the true accuracy of the diagnostic procedures tested in VAP patients is unpredictable.

Nevertheless, Michaud and coworkers [7] found that patient selection criteria have the greatest impact on evaluation of a test's performance in VAP patients. Moreover, heterogeneity among diagnostic criteria and the lack of a gold standard for VAP diagnosis that could be applied across the various diagnostic studies may lead to several biases. Previous antibiotic exposure and technical aspects of the diagnostic tests used may also have an effect on reported accuracy. Souweine and coworkers [8] recommended use of lower thresholds on quantitative cultures in patients who have recently started antibiotic therapy. However, whether

ATS = American Thoracic Society; BAL = bronchoalveolar lavage; COPD = chronic obstructive pulmonary disease; IDSA = Infectious Disease Society of America; PSB = protected specimen brush; QEA = quantitative endotracheal aspirate; VAP = ventilator-associated pneumonia. 
thresholds maintain their discriminatory value in such patients requires evaluation. Until proven otherwise, a strategy to rule in or rule out the presence of pneumonia based on a bacterial count threshold has not been adequately tested and should not be routinely used to make decisions regarding antimicrobial treatment.

Whether use of quantitative cultures of lower respiratory tract secretions has a diagnostic role to play remains controversial. ATS/IDSA guidelines [2] recommend that quantitative cultures should be performed on endotracheal aspirates or samples collected bronchoscopically, and the choice of method depends on local expertise, experience, availability and cost. However, Heyland and coworkers [9] recently reported, in a randomized multicentre clinical trial, that an invasive bronchoscopy based strategy and a noninvasive nonquantitative endotracheal aspirate culture based one were associated with similar clinical outcomes and antibiotic use.

The best available evidence suggests that the clinical data are insufficient to establish a definite diagnosis of pneumonia. Culture of pulmonary secretions may help to refine the physician's clinical suspicion [10], but it should not be used alone to confirm or refute it. Microbiologic findings can help to tailor the antibiotic spectrum in selected patients, although they should not be considered alone but rather with reassessment of clinical response at 48 to 72 hours after onset of pneumonia [11].

Albert Einstein (1879-1955) once stated that, 'Things should be made as simple as possible, but not simpler'. The dualism of colonization versus infection that results from use of a single cut-off to define pneumonia may represent an oversimplification of a complex phenomenon. Exclusion of the presence of pneumonia based simply on a 'magic' number, as a simpler way to approach diagnosis, is a dangerous policy. We should avoid being caught in this trap, because by oversimplifying the diagnostic process in this way we take on significant risk for mistreating (over or under) our patients. Efforts should be focused on implementing bundles of measures based on best evidence for diagnosis and treatment, integrating clinical features and microbiology data, in order to obtain a simple and effective approach to management of pneumonia.

\section{Competing interests}

The authors declare that they have no competing interests.

\section{Acknowledgement}

Supported by Fondo de Investigaciones Sanitárias (PI05/2410), CIBERes Enfermedades Respiratórias (CIBER 06/06/0036) and MARATOTV3.

\section{References}

1. El Solh AA, Akinussi ME, Pineda LA, Mankowski CR: Diagnostic yield of quantitative endotracheal aspirates in patients with severe nursing-home acquired pneumonia. Crit Care 2007, 11:R57.
2. American Thoracic SocietyL Guidelines for the management of adults with hospital-acquired, ventilator-associated, and healthcare-associated pneumonia. Am J Respir Crit Care Med 2005, 171:388-416.

3. Gallego M, Rello J: Diagnostic testing for ventilator-associated pneumonia. Clin Chest Med 1999, 20:671-679.

4. Fagon JY, Rello J: Targeted antibiotic management of ventilator-associated pneumonia. Clin Microbiol Infect 2006, Suppl 9: 17-22.

5. Zou KH, O'Malley AJ, Mauri L: Receiver-operating characteristic analysis for evaluating diagnostic tests and predictive models. Circulation 2007, 115:654-657.

6. Irwig L, Bossuyt P, Glazsiou P, Gatsonis C, Lijmer J: Evidence base of clinical diagnosis: designing studies to ensure that estimates of test accuracy are transferable. BMJ 2002, 324: 669-671.

7. Michaud S, Suzuki S, Harbarth S: Effect of design-related bias in studies of diagnostic tests for Ventilator-associated pneumonia. Am J Respir Crit Care Med 2002, 166:1320-1325.

8. Souweine B, Veber B, Bedos JP, Gachot B, Dombret MC, Regnier B, Wolff M: Diagnosis accuracy of protected specimen brush and bronchoalveolar lavage in nosocomial pneumonia: impact of previous antimicrobial treatments. Crit Care Med 1998, 26:236-244.

9. The Canadian Critical Care Trials Group: A randomized trial of diagnostic techniques for ventilator-associated pneumonia. $N$ Engl J Med 2006, 355:2619-2630.

10. Klompas $\mathrm{M}$ : Does this patient have ventilator-associated pneumonia? JAMA 2007, 297:1583-1593.

11. Vidaur L, Gualis B, Rodriguez A, Ramirez R, Sandiumenge A, Sirgo G, Diaz E, Rello J: Clinical resolution in patients with suspicion of ventilator-associated pneumonia: a cohort study comparing patients with and without acute respiratory distress syndrome. Crit Care Med 2005, 33:1248-1253. 